\title{
Next-generation RNA sequencing reveals differential expression of MYCN target genes and suggests the mTOR pathway as a promising therapy target in $M Y C N$-amplified neuroblastoma
}

\author{
Alexander Schramm ${ }^{1}$, Johannes Köster ${ }^{1,2}$, Tobias Marschall ${ }^{3,4}$, Marcel Martin ${ }^{4}$, Melanie Schwermer ${ }^{1}$, Kathrin Fielitz ${ }^{1}$, \\ Gabriele Büchel ${ }^{1}$, Matthias Barann ${ }^{5}$, Daniela Esser ${ }^{5}$, Philip Rosenstiel ${ }^{5}$, Sven Rahmann ${ }^{2,4}$, Angelika Eggert ${ }^{1}$ \\ and Johannes H. Schulte ${ }^{1}$ \\ ${ }^{1}$ Department of Pediatric Hematology and Oncology, University Children's Hospital, Essen, Germany \\ ${ }^{2}$ Department of Genome Informatics, University Hospital Essen, Essen, Germany \\ ${ }^{3}$ CWI Amsterdam, Statistical Modeling and Comparative Genomics Group, Amsterdam, The Netherlands \\ ${ }^{4}$ Department of Computer Science, LS11, University of Dortmund, Dortmund, Germany \\ 5 Institute of Clinical Molecular Biology, Christian Albrechts University, Kiel, Germany
}

In many cancer types, MYC proteins are known to be master regulators of the RNA-producing machinery. Neuroblastoma is a tumor of early childhood characterized by heterogeneous clinical courses. Amplification of the MYCN oncogene is a marker of poor patient outcome in this disease. Here, we investigated the MYCN-driven transcriptome of 20 primary neuroblastomas with and without MYCN amplification using next-generation RNA sequencing and compared the results to those from an in vitro cell model for inducible MYCN (SH-EP MYCN-ER). Transcriptome sequencing produced 30-90 million mappable reads for each dataset. The most abundant RNA species was mRNA, but snoRNAs, pseudogenes and processed transcripts were also recovered. A total of 223 genes were significantly differentially expressed between $M Y C N$-amplified and single-copy tumors. Of those genes associated with MYCN both in vitro and in vivo, 32\% of MYCN upregulated and $37 \%$ of MYCN downregulated genes were verified either as previously identified MYCN targets or as having MYCN-binding motifs. Pathway analyses suggested transcriptomal upregulation of mTOR-related genes by MYCN. MYCN-driven neuroblastomas in mice displayed activation of the mTOR pathway on the protein level and activation of MYCN in SH-EP MYCN-ER cells resulted in high sensitivity toward mTOR inhibition in vitro. We conclude that next-generation RNA sequencing allows for the identification of MYCN regulated transcripts in neuroblastoma. As our results suggest MYCN involvement in mTOR pathway activation on the transcriptional level, mTOR inhibitors should be further evaluated for the treatment of $M Y C N$-amplified neuroblastoma.

Neuroblastoma (NB) is a cancer of childhood presenting with extreme courses: while aggressive NB still poses a challenge to clinicians and recurrent disease renders an infaust prognosis despite multimodal therapy, ${ }^{1}$ intermediate-risk patients with favorable tumor biology can be successfully treated with a less intensive treatment. ${ }^{2}$ Moreover, benign neuroblastic tumors can be cured by surgery combined with a "wait-and-see" strategy. ${ }^{3}$ Many efforts have been under-

Key words: neuroblastoma, next-generation sequencing, MYCN, mTOR pathway

Additional Supporting Information may be found in the online version of this article.

Grant sponsor: Deutsche Forschungsgemeinschaft (DFG);

Grant number: SFB 876

DOI: $10.1002 / \mathrm{ijc} .27787$

History: Received 19 Mar 2012; Accepted 31 Jul 2012; Online 21 Aug 2012

Correspondence to: PD Dr. Alexander Schramm, Department of Pediatric Hematology and Oncology, University Children's Hospital Essen, Hufelandstr. 55, 45122 Essen, Germany, Tel.: +49-201-7232506, Fax: +49-201-7235750, E-mail: alexander.schramm@uni-due.de taken to identify risk factors for aggressive neuroblastoma, but amplification of the MYCN oncogene is still the most powerful single predictor of adverse outcome of NB. ${ }^{4}$ MYCN amplification is observed in about $20 \%$ of all NB patients and is usually associated with fatal outcome of the disease. ${ }^{5}$ As MYCN is a transcription factor, thus not representing an ideal druggable target, molecular analyses of this subgroup of NB promises to result not only in a better understanding of MYCN driven tumor biology but also in novel treatment options for this most aggressive tumor subtype.

With the onset of affordable next-generation sequencing (NGS) technologies, it can be anticipated that underlying gene mutations for every tumor will be identified within the next few years. For NB, first reports on the mutational spectrum of the disease have been recently published, ${ }^{6}$ stressing the importance of MYCN, chromosomal stability and mutations in neuritogenesis genes for neuroblastoma development. In neuroblastoma, a genetic association with the onset of the disease has only been found for a small fraction of all cases. Activating mutations in the coding gene for anaplastic lymphoma kinase (ALK) have been described in about eight percent of all NB patients, ${ }^{7-11}$ while mutations in the 


\section{What's new?}

In neuroblastoma, amplification of the MYCN oncogene is associated with a poor prognosis. Now that next-generation sequencing technologies are becoming more affordable, it may soon be possible to identify the mutations underlying individual tumors, and to use that information to develop individualised treatment strategies. In this study, the authors used "RNA deep sequencing" to examine the MYCN-driven transcriptomes of a number of neuroblastomas. Their results suggest that mTOR inhibitors should be further evaluated for the treatment of $M Y C N$-amplified neuroblastoma.

PHOX2B gene, which is also associated with Hirschsprung disease, are even less frequent $\left(1-2 \%^{12,13}\right)$. Therefore, the vast majority of neuroblastomas are sporadic cases with a heterogeneous mutational spectrum. In the light of endeavors to treat patients according to their individual risk profile, these heterogeneity could imply that risk factors such as $M Y C N$ amplification or loss of chromosome $11 \mathrm{q}^{14,15}$ will still have an impact on the choice of individualized treatment. Moreover, the impact of the "mutanome" as the sum of all cancer-associated genetic changes on transcriptomal alterations remains to be explored.

We have previously reported on tumor-associated changes in the small RNA transcriptome of primary neuroblastoma thus describing the entire spectrum of NB-associated miRNAs. ${ }^{16}$ The oncogenic or tumor-suppressive functions of these miRNAs could be verified by independent methods and experiments ${ }^{17,18}$ exemplifying that the results from next-generation sequencing can be meaningfully exploited for functional studies.

Here, we focused on the coding transcriptome of primary NB by comparing absolute RNA expression levels in tumor samples from patients with and without MYCN amplification using next-generation RNA sequencing. To identify direct MYCN targets we also included an NB cell line with inducible induction of MYCN in the sequencing pipeline. The intersection of regulated genes between the in vivo and the in vitro samples was anticipated to include genes, whose expression is tightly linked or directly regulated by MYCN. With this information at hand it was expected that MYCN gene regulatory networks as well as potential targets for MYCNdriven NB could be identified.

\section{Material and Methods}

\section{Cell culture and in vitro analyses}

Cultivation of human neuroblastoma cells SH-EP, SH-EP MYCN-ER and WAC2 was performed as previously described. ${ }^{19}$ SH-EP is a MYCN null neuroblastoma cell line and genetically identical to SH-SY5Y but displaying an epithelium-like phenotype. ${ }^{20}$ WAC2 is a subclone of SH-EP engineered to stably overexpress $\mathrm{MYCN}^{21}$ Activation of MYCN in SH-EP MYCN-ER was achieved using 4-hydroxy tamoxifen and confirmed after $72 \mathrm{hr}$ by detecting transcriptional induction of the MYCN-target gene, ODC-1, as described. ${ }^{19}$ Cell viability following treatment with BEZ-235 (Axon Medchem, Groningen, The Netherlands) was measured using MTT (3-(4,5-dimethylthiazol-2-yl)-2,5-diphenyltetrazoliumbromide) as previously described. ${ }^{22}$

\section{RNA preparation for next generation sequencing}

NB patients included in this study were from German trials NB 97 or 2004, respectively. Representative areas of histologically confirmed, snap-frozen NB were cut on dry ice. No preselection for tumor cell fraction or microenvironment was performed. Total RNA extraction of NB tumor samples and cell lines described above was performed by silica gel-based membrane purification methods (RNeasy Mini kit or MicroRNeasy kit, Qiagen, Hilden, Germany) according to the manufacturer's instructions. Depletion of ribosomal RNAs was achieved using the RiboMinus Human Transcriptome Kit (Invitrogen, Carlsbad, CA) according to the manufacturer's instructions. RNA quality as well as rRNA depletion efficiency was controlled by capillary gel electrophoresis using the Bioanalyser 2100 (RNA nano kits, Agilent).

\section{Library generation and transcriptome sequencing}

Library preparation was performed as previously described. ${ }^{23}$ Of the depleted rRNA, 50-500 ng was used as input for the whole transcriptome analysis kit (Applied Biosystems, Carlsbad, CA). Library preparation was performed according to the manufacturer's protocol. Sequencing was performed using the SOLiD “Top Paired End Seq DNA Frag Kit MM 50/35 v4" (Applied Biosystems, Carlsbad, CA) on the SOLiD V4 platform. In total 24 libraries were generated and sequenced.

\section{Sequence processing and mapping}

SOLiD reads were mapped to the Human Genome RefSeq Hg19 using Bioscope v1.21. A clearzone of 5.0 and mismatch penalty of -2.0 were set, and a repetitive mapping was performed using the following settings: 38.3.0, 25.2.0 (anchorLength.mismatchAllowed.anchorStart). See the "Bioscope Userguide" for a more detailed description of the Bioscope mapping algorithm. For ambiguous mappings, only the alignment with best mapping quality was considered. Based on the ENSEMBL Hg19 annotation (rev. 63), per gene expression values were set as the maximum coverage of the exonic gene regions.

\section{Normalization}

Gene expressions were normalized to allow comparison across datasets. A qq-scale normalization as described in Ref. ${ }^{16}$ was performed separately for the tumor samples and the in vitro datasets. Quantile-quantile (qq) plots of the distribution of gene expression values of each dataset against one arbitrarily chosen reference dataset were compared. By 
considering only the $75-95 \%$ quantiles, robustness against less abundant genes and outliers was ensured. With the scaling factor given by the median of differences between these quantiles, a purely linear transformation was performed. ${ }^{16}$

\section{Statistical testing and cluster analysis}

Statistical analysis and clustering of gene expressions was performed using R 2.14 (http://www.r-project.org). Genes were tested for differential expression using MYCN status (amplified vs. normal) or survival (see Supporting Information Table 1 for patient characteristics) as variables. For each gene, the fold change was computed as the ratio of the mean gene expressions of the classes, while fold changes between 1 and 0 were replaced by the negative of their inverse. In the tumor datasets, genes with a $p$ value $<0.05$ and an absolute fold change of at least 1.5 were considered to be significantly differentially expressed. The false discovery rate (FDR) was calculated using Benjamini-Hochberg multiple testing correction using the $\mathrm{R}$ library "multtest."24,25 Hierarchical clustering was performed based on Canberra distances. ${ }^{16}$

\section{In silico verification of potential MYCN targets}

A list of potential MYCN targets was obtained by selecting genes that were significantly differentially expressed using MNA classification in the tumor and exhibited an absolute fold change of at least 1.5 in the in vitro datasets. Potential targets were verified using existing databases (MYC target gene database, http://www.myccancergene.org, ${ }^{26}$ and the MYCNot Database [http://medgen.ugent.be/MYCNot]). Further, the MYCN binding motif E-Box (CACGTG), together with its isoforms (CATGTG, CATTTG, CATCTG, CAACTG) reported by Ref. ${ }^{27}$ was tested for significant overrepresentation $(p \leq 0.05)$ in the promoter regions of the genes. Therefore, each motif was tested in a 1,000 base region upstream of the first exon of each gene using the sequence alignment tool MosDi (http://code.google.com/p/mosdi $/,{ }^{28}$ ).

\section{Confirmation of mTOR pathway activation in tumors from TH-MYCN mice}

TH-MYCN mice were housed in the central animal facility of the University Hospital Essen. These mice developed neuroblastoma at a frequency comparable to published data. ${ }^{29}$ Total protein was isolated from tumors and normal livers of three tumor-bearing animals. Western blot analysis was performed to detect total Akt, phospho-Akt, total p70S6K and phospho-p70S6K to evaluate Akt/mTOR pathway activation. All primary antibodies were obtained from Cell Signaling (Beverly, MA).

\section{Results}

Following RNA isolation and rRNA depletion, transcriptomes from 20 primary NB with and without MYCN amplification and a NB cell line with and without induction of MYCN (SH-EP MYCN-ER) were analyzed by NGS using paired-end sequencing on a SOLiD4 sequencer (Applied Biosystems).

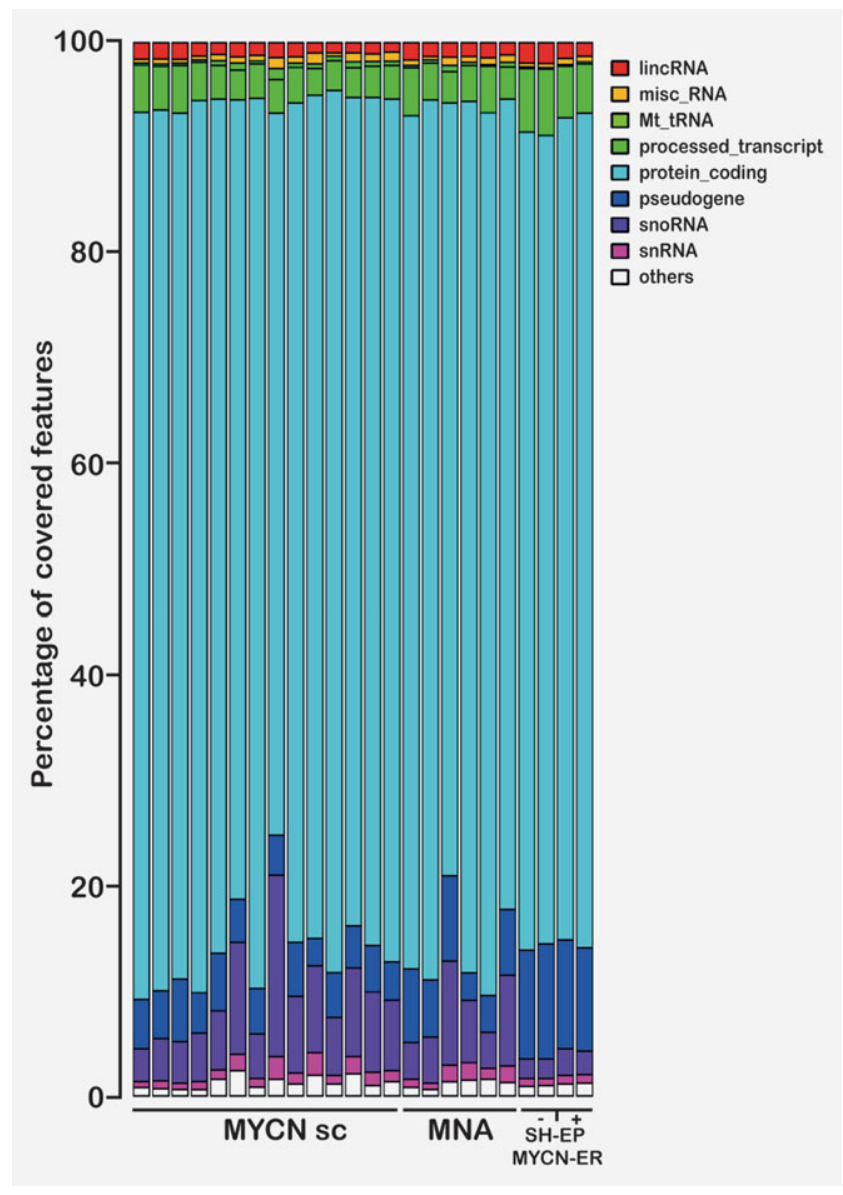

Figure 1. Distribution of RNA species for the samples sequenced by NGS. Note that the predominant form is mRNA (light blue), but that also lincRNAs, snoRNAs and snRNAs were readily detectable, although at lower levels. Immunoglobulin pseudogenes and miRNAs (subsumed in white) are only present in minor amounts due to the size selection and depletion strategy. "MYCNsc" and "MNA" refers to samples from MYCN single-copy or MYCNamplified patients, respectively. "SH-EP MYCN-ER" refers to samples from the SH-EP MYCN-ER cell line before ("-") and after induction of MYCN ("+") by 4-OHT. Transcriptomes of cell lines were analyzed in duplicate from independent biological samples.

Patient characteristics are listed in Supporting Information Table 1. Mapping against the human genome RefSeq Hg19 was performed using BioScope (see Methods). On average, 31.615.883 reads for each tumor, and 89.689.520 reads for each in vitro dataset could be mapped to the reference genome. The most abundant class of RNA species were protein coding mRNAs, followed by snoRNAs, pseudogenes and processed transcripts, which are noncoding transcripts without an open reading frame (Fig. 1). The distribution of RNA species underscores both the efficiency for rRNA depletion and mRNA enrichment in our samples.

All transcripts were mapped to the ENSEMBL Hg19 annotation containing 53,717 features including coding genes. Of those, 8030 coding genes were found to have absolute expression counts $>10$ in at least five samples. To obtain comparable expression values, a qq-scale normalization 




Figure 2. Effects of normalization on transcriptome comparability. A qq-normalization was performed separately for in vivo (a) and in vitro (b) samples, using the quantiles between 75 and $95 \%$ to ensure robustness. Histogram of uncorrected $p$ values after testing equality of expression count means for each RNA between MYCN-amplified vs. MYCN single copy tumors (c) or patients who died from their disease vs. patients who survived (d). Distribution of P-values is expected to be uniformly distributed across the unit interval (blue line), when data with equal means were compared. Here, $p$ values $<0.05$ (red line) are enriched, indicating significant differences between the groups analyzed.

among all tumor datasets was performed (see methods, Figs. $2 a$ and $2 b$ ) and expression values were then calculated for all tumor datasets (see methods). We first focused on differential expression analysis to find gene expression patterns as well as individual genes with significant expression differences between $M Y C N$-amplified and $M Y C N$ single copy tumors or expression patterns associated with survival. Only genes with an absolute expression count of at least 10 in at least five tumor datasets were selected for these analyses. Based on our filter criteria, 945 and 727 genes were used for classification according to MYCN status or survival, respectively, and $p$ values for differential expression were calculated. A total of 223 genes were significantly differentially expressed between patients with MYCN amplification and patients with normal MYCN status. For patients surviving their disease for $>3$ years without an event, 211 genes were found to be significantly different when compared to the patients succumbing to their disease. To address the problem of multiple testing, we plotted the distribution of $p$ values to evaluate if low $p$ values were over-represented between the different groups. Indeed, there is an enrichment of low $p$ values when gene expression was compared for patients with or without MYCN amplification and those surviving the disease to those who succumbed (Figs. $2 c$ and $2 d$ ). This finding implies that there are true transcriptomal differences between these groups. Looking at the most differentially expressed transcripts con- sidering MYCN amplification (Fig. 3a) or survival (Fig. 3b) both upregulation and downregulation of candidate MYCN targets could be detected. Mostly, we observed regulation of protein coding mRNAs, but also differential expression of snoRNAs including SNORA71C and SNORD116 was observed. An unsupervized hierarchical clustering using all significantly differentially expressed genes resulted in a perfect separation between MYCN amplified and MYCN normal patients (Fig. 3c). When trying to separate patients according to survival based on the list of significantly differentially expressed transcripts, we noticed that there was one misclassification (Fig. 3d). Taken together, the principled differences between the transcriptomes of aggressive and less aggressive neuroblastomas allowed for the separation of these clinically divergent groups. Genes and transcripts with significant differential expression dependent on the MYCN amplification status are candidate direct or indirect MYCN targets. To obtain insights into a direct MYC regulation of the transcripts that were identified in the primary tumors, a wellestablished model system of inducible MYCN function in a neuroblastoma cell line, SH-EP MYCN-ER, was used. In this model system, MYCN is expressed in a cell line without endogenous MYCN as a fusion gene with the estrogenresponsive domain of the ER receptor. MYCN activation can only be induced in the presence of 4-hydroxy-tamoxifen causing a nuclear translocation of the MYCN-ER fusion 

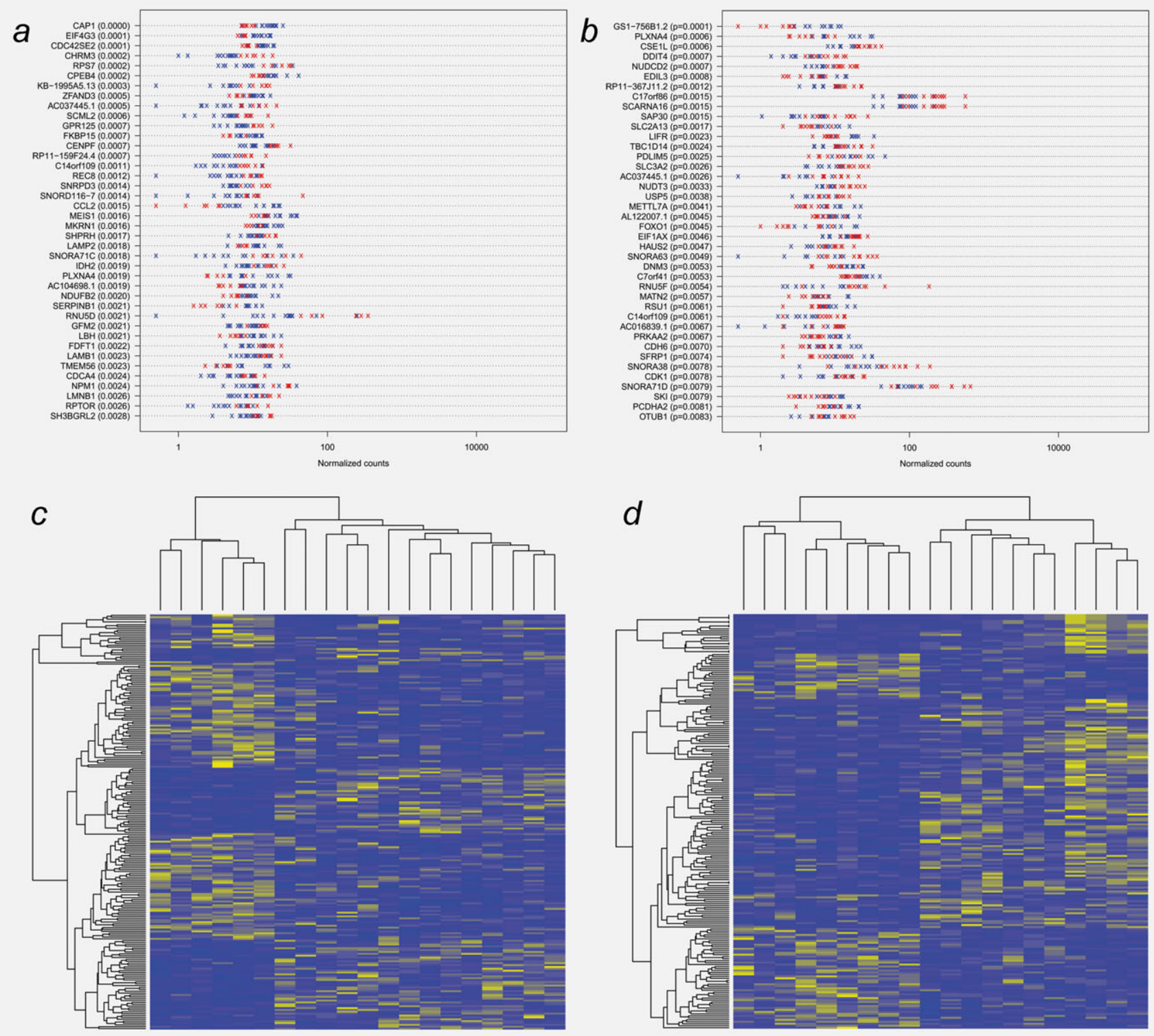

Figure 3. Significantly different expressed genes in tumor samples grouped by MYCN status (a) or survival (b). The 40 genes with the lowest uncorrected $p$ values are presented as strip chart of normalized read count. Blue crosses represent expression values in MYCN single copy (a) and surviving patients (b), respectively. Red crosses depict expression values in $M Y C N$-amplified $(a)$ and deceased patients $(b)$, respectively. Heat map and cluster dendrogram of all significant $(p<0.05)$ RNAs differentially expressed between MYCN-amplified vs. MYCN single copy patients and surviving patients vs. deceased, respectively. Note that the MYCN status allows for perfect separation, while the classification according to survival yields one wrongly assigned sample. Clustering was based on Canberra distance and single-linkage clustering.

protein. The transcriptome of SH-EP MYCN-ER cells with or without induction of functional MYCN was analyzed by RNA sequencing as described for the primary tumors and differentially expressed transcripts in the presence or absence of functional MYCN were identified accordingly. The list of transcripts associated with MYCN amplification in vivo and transcripts induced by MYCN in vitro were then compared to narrow down the candidate genes directly targeted by MYCN. For this purpose, we first omitted all genes that had a fold change (either up or down) of $<1.5$ in vitro. Second, we kept only those genes, for which the sign of the fold change (i.e., the direction of regulation) was the same in tumors and in vitro. Application of these filter criteria resulted in 25 genes that were upregulated (positive fold change) and 19 genes that were downregulated (negative fold change) upon MYCN induction in vitro (Figs. $4 a$ and Supporting Information S2) and MYCN amplification (Figs. $4 b$ and Supporting Information S2). Among the upregulated genes, we found three known MYCN targets as described in publicly available databases (see Methods). Interestingly, we also identified 7 MYCN target genes to be downregulated. Homology searches identified an E-Box binding motif, which is the MYCN transcription factor binding site, in the promoter region of five upregulated genes and one 

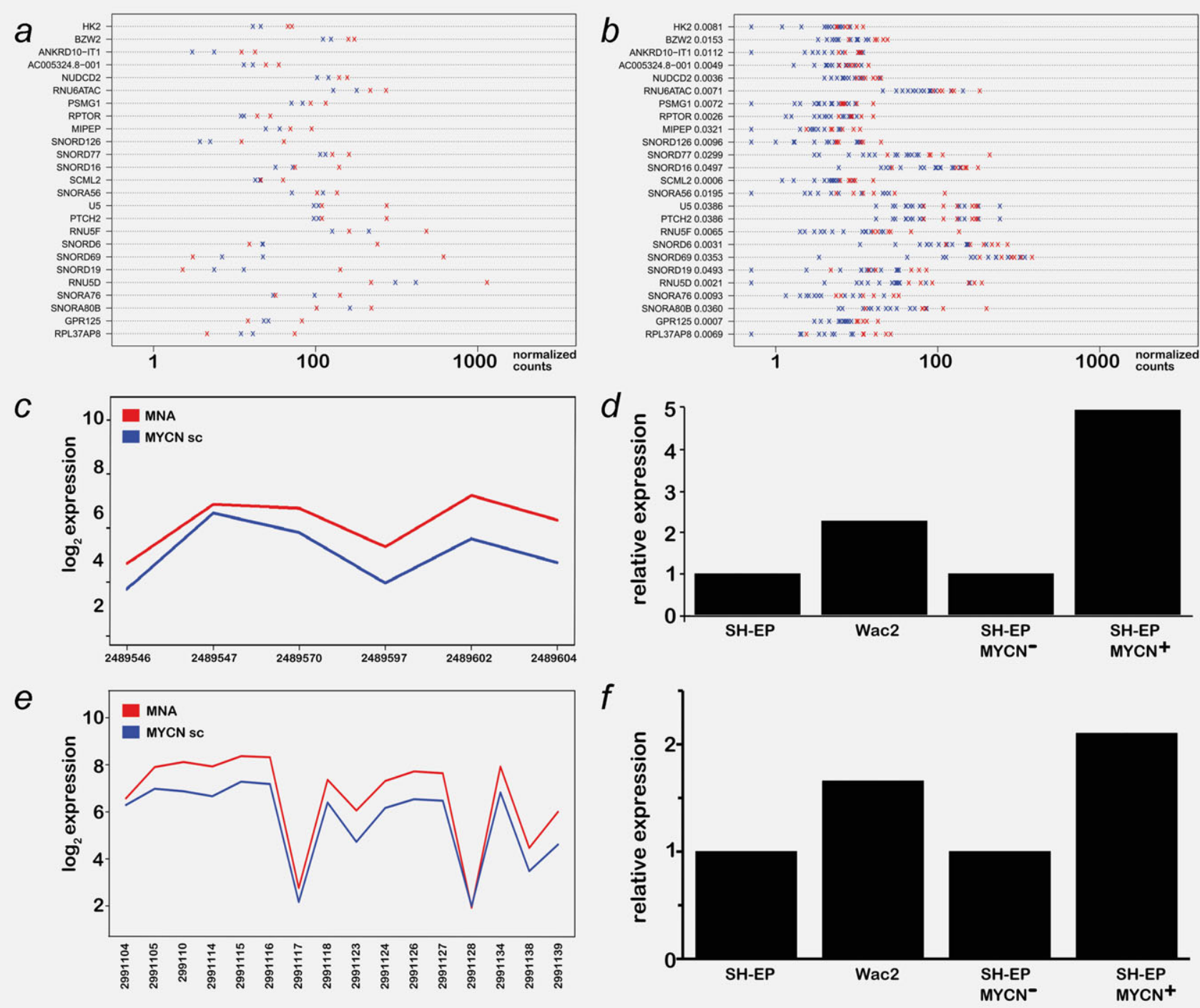

Figure 4. Significantly differentially expressed genes between $M Y C N$-amplified tumors and $M Y C N$ normal tumors verified in an in vitro NB model with inducible MYCN expression. Data are presented as strip charts of normalized read count as pairwise comparison of genes that are upregulated upon MYCN induction in vitro (a) and positively correlated with MYCN amplification in vivo (b). Blue color depicts data from the NB in vitro system without MYCN induction (a) or from MYCN normal tumors $(b)$. Red color depicts data from the NB in vitro system with MYCN induction (a) or from tumors with MYCN amplification (b). The top target genes HK2 and BZW2 are upregulated in $M Y C N$-amplified tumors in an independent cohort of primary NB analyses using exon-level expression analyses (c, e, Schramm et al., submitted). Numbers refer to the probe sets for the respective genes on the array. HK2 and BZW2 mRNA are also upregulated in two in vitro NB models, upon overexpression or induction of MYCN, respectively $(d, f)$. Displayed are the fold changes of normalized HK2 and BZW2 mRNA expression measured by semi-quantitative RT-PCR in MYCN-overexpressing WAC2 cells compared to the parental cell line SHEP and in SH-EP MYCN-ER cells following MYCN activation (SH-EP MYCN+) compared to cells without induction of MYCN (SH-EP MYCN-).

downregulated gene (Table 1 , see Methods), implying that these genes are also bonafide direct MYCN targets. In total, $32 \%$ of the upregulated and $37 \%$ of the downregulated genes could be verified as either known MYCN targets or novel targets containing E-boxes. We first validated upregulation of the top-ranked target genes hexokinase 2 (HK2) and BZW2 in the SH-EP MYCN-ER model system as well as in a SH-EP subclone, designated WAC2, which is engineered to stably overexpress $\mathrm{MYCN}^{21}{ }^{21}$ Upregulation of $H K 2$ as well as BZW2 mRNA by real-time PCR could be seen in both of the in vitro systems with forced expression of MYCN. HK2 and BZW2 expression were also found to correlate with $M Y C N$ amplification in an independent cohort of 113 neuroblastoma patients profiled by Affymetrix HuEXST1.0 arrays (Schramm et al., submitted, Figs. $4 c$ and $4 e$ ). Taken together, reanalyses of microarray data from primary NB confirmed that $H K 2$, BZW2 and RPTOR are significantly correlated with MYCN amplification (Supporting Information Fig. 2). These data exemplify that the MYCN targets identified here by sequencing can also be validated by independent methods. We next 
Table 1. Intersection of MYCN targets identified both in vitro and in vivo

\begin{tabular}{lll}
\hline Database/Motif & \multicolumn{1}{c}{$\begin{array}{c}\text { Upregulated } \\
\text { MYCN targets }\end{array}$} & $\begin{array}{c}\text { Downregulated } \\
\text { MYCN targets }\end{array}$ \\
\hline MYCNot & HK2 & ITM2C \\
MycDB & U5-RNA & MEIS1 \\
& SCML2 & LAMP2 \\
& HK2 & GABARAP \\
& & SERPINB1 \\
\hline E-Box overrepresented & SNORA76 (CACGTG) & CD44 (CATGTG) \\
& & \\
& & \\
& SNORD6 (CATGTG) & \\
& SNORD77 (CATTG) & \\
& PTCH2 (CATTG) & \\
& SCML2 (CATCTG) & \\
\hline
\end{tabular}

focused on classification of MYCN targets genes to better understand the molecular processes regulated by MYCN in neuroblastoma. For these purpose, we looked into GO and KEGG databases to identify pathways significantly associated with MYCN. Analyses of over-representation of genes within a pathway indicated that MYCN significantly modulated genes involved in protein biosynthesis and the mTOR pathway. GO analyses revealed that genes coding for ribosomal subunits, as well as genes involved in "ribonucleoprotein complexes" were significantly enriched and upregulated both in vitro and in vivo by MYCN (Fig. $5 a, p$ value <0.001). KEGG pathway analyses confirmed this association and implied that three genes of the mTOR pathway, REDD1/ DDIT4, 4EBP1 and RPTOR, were significantly altered by the MYCN status in vivo and in vitro (Fig. $5 b$ and Supporting Information Table 2 , adjusted $p$ value: $8 \times 10^{-4}$ ). A significant correlation between RPTOR expression and MYCN amplification could also be seen in an independent panel of primary neuroblastoma (Supporting Information Fig. 2). We therefore decided to look for mTOR pathway activation in a model of MYCN-driven neuroblastoma, the TH-MYCN mouse. ${ }^{29}$ In this model system, we used phosphorylation of the S70 kinase and $\mathrm{AKT}$ as a readout of $\mathrm{AKT} / \mathrm{mTOR}$ activation in the MYCN-driven tumors. Indeed, we could demonstrate tumorspecific activation of the mTOR pathway in tumors obtained from TH-MYCN mice compared to normal tissue from the same mice (Fig. $5 c$ ). To test, whether upregulation of mTOR pathway genes was concomitant with sensitivity to mTOR inhibition, we performed in vitro proliferation assays using the mTOR inhibitor BEZ-235, which is currently in late phase of clinical development. Sensitivity to BEZ-235 was comparable between SH-EP and WAC2 cells and even more pronounced in SH-EP MYCN-ER cells upon MYCN activation (Fig. 5d, SH-EP MYCN+).Thus, we could link two cancer-relevant pathways, protein biosynthesis and the mTOR pathway to the MYCN status in neuroblastoma by using transcriptome NGS.

\section{Discussion}

Deciphering cancer genomes and transcriptomes is estimated to yield a wealth of information, which can be exploited for modeling tumorigenesis as well as for setting up individualized treatment of cancer patients. Reports available so far have established a correlation between mutational frequency in cancer, patient age and exposition to potentially carcinogenic agents such as UV-light or smoking. This complicates the hunt for the driving mutational events given a high background of acquired passenger mutations. Moreover, intratumoral heterogeneity could further complicate the design of rationale, individualized treatment options. ${ }^{30}$ In those childhood tumors, for which entire genomes have been sequenced, such as medulloblastoma, ${ }^{31}$ retinoblastoma $^{32}$ and neuroblastoma, ${ }^{6}$ patients have significantly lower numbers of mutations compared to adult tumors. This should in principle facilitate the search for the tumor driving events, at least for the sporadic forms of the disease. Still, the route from the tumor genome to the tumor phenotype demands analyses of the intermediate steps including alterations in the tumor transcriptome. RNA profiles can be expected not only to include signatures but also to give valuable hints on aberrantly activated signaling pathways in tumors, which can be used for targeted therapies. As a first step toward this direction, we here describe for the first time the entire transcriptome of 20 primary $\mathrm{NB}$ using next-generation sequencing. Because we previously described the non-coding transcriptome of $\mathrm{NB},{ }^{16}$ we here focused on protein-coding transcripts and identified genes and patterns associated with the most aggressive NB subtype, characterized by amplification of the MYCN oncogene. As expected, mRNA was identified as the predominant RNA species following annotation of sequences obtained by RNA-NGS, but we could also detect a considerable amount of snoRNAs, whose function is presently poorly understood. Previous reports have also linked expression of ncRNAs/snoRNAs as well as miRNAs to MYCN amplification ${ }^{19,33}$ and it was shown that MYCN expression itself is controlled by miRNAs. ${ }^{34}$ It is speculated that ncRNAs can function in regulating gene expression, which is also of functional importance in developmental processes as well as in cancer progression. ${ }^{35-37}$ Thus, the noncoding part of the transcribed regions of the genome can be expected to harbor an additional layer of regulation to gene expression in addition to miRNAs in neuroblastoma and other tumors.

Analyses of the most divergently expressed transcripts between $M Y C N$-amplified and $M Y C N$-single copy tumors confirmed the principled differences between these two groups, which has also been pointed out before. ${ }^{38,39}$ Hierarchical clustering using these differentially expressed transcripts as input revealed a perfect separation of patients with and without MYCN amplification, emphasizing the homogeneity of discriminative patterns (Fig. $3 c$ ).

Although the sequencing depth achieved in our approach was not sufficient to cope with alternative transcript use, we 
a

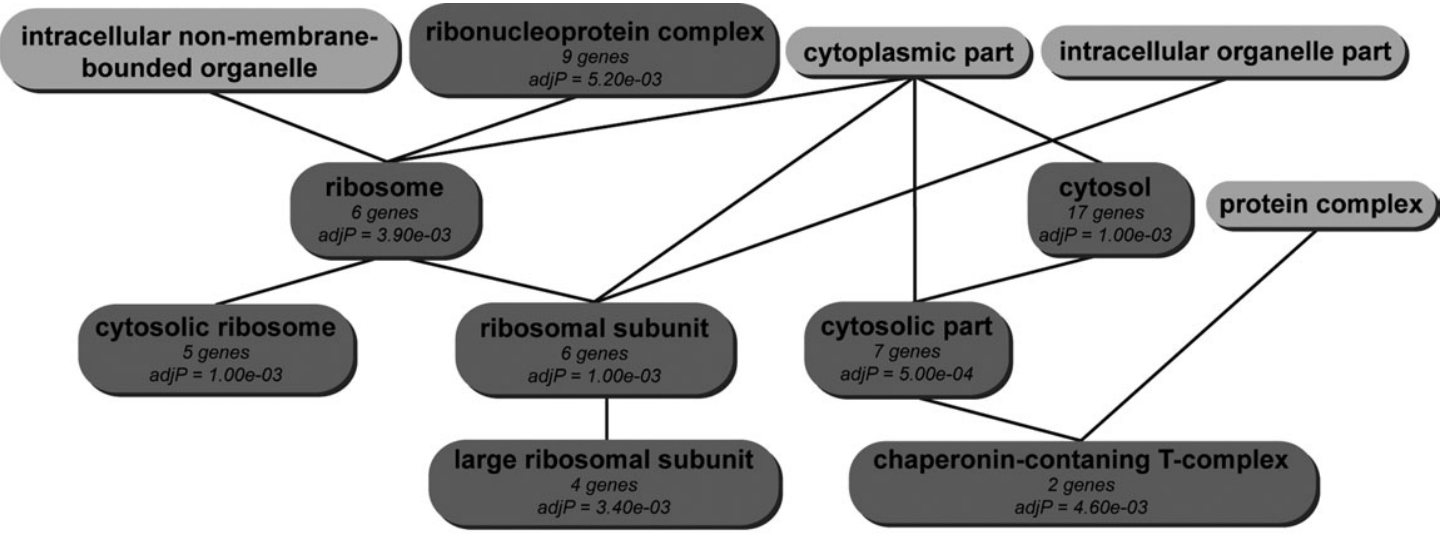

$b$
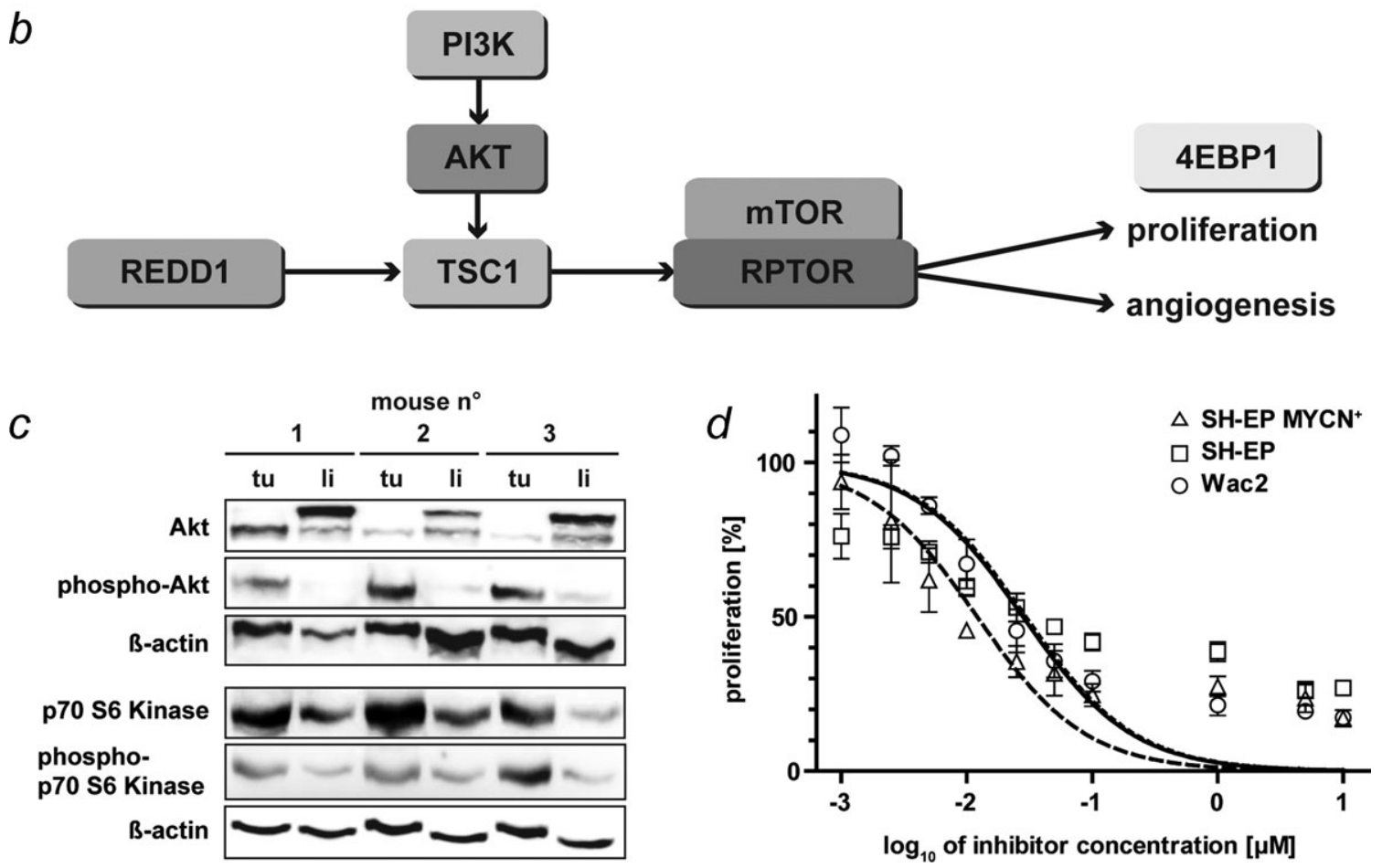

Figure 5. (a) Gene ontology (GO) analyses revealed a significant association of genes upregulated in tumors from patients with MYCN amplification with GO terms for protein biosynthesis (marked in dark grey). (b) KEGG pathway analyses returns a significant association of genes upregulated in tumors from patients with MYCN amplification with the mTOR pathway (genes marked in dark grey are upregulated in MYCN-amplified tumors). (c) Activation of Akt/mTOR pathway is seen in tumors (tu) from MYCN-driven murine neuroblastoma but not in control tissue (liver = li). (d) Sensitivity to mTOR pathway inhibition using BEZ-235 is high in neuroblastoma cells and comparable between parental SH-EP cells (open squares, IC50 = 29 nM) compared to those with enforced expression (WAC2, open circles, IC50 = $28 \mathrm{nM}$ ) with slightly increased sensitivity upon activation of MYCN (SH-EP MYCN+, open triangles, IC50 = $12 \mathrm{nM}$ ).

were able to identify differentially expressed transcripts associated with MYCN in vivo and regulated by $\mathrm{MYCN}$ in vitro in a NB model system with inducible MYCN (Fig. 4). The most prominently regulated target, $H K 2$, was validated both in an independent cohort of primary neuroblastomas as well as in an NB in vitro model with inducible nuclear MYCN translocation, SH-EP MYCN-ER. Induction of HK2 as well as other targets of the glycolytic pathway has been recently associated with the MYCN-mediated Warburg effect in neuroblastoma. ${ }^{40}$ Functional significance of other targets, especially the snoRNAs, which are upregulated both in vitro and in vivo by MYCN remains to be determined.

Analyses of associated pathways using GO and KEGG databases linked protein biosynthesis and the mTOR pathway to MYCN-upregulated genes in NB and these were also positively correlated with $M Y C N$ amplification in primary tumors. Protein biosynthesis has also been reported to be induced by MYCN in vitro before in an unbiased approach using SAGE technology ${ }^{41}$ and this was verified in this study also for primary tumors. In a previous array based study, 
cell cycle genes and genes involved in kinetochore assembly had been associated with survival and $M Y C N$ amplification in primary NB, but these genes have not been verified using $\mathrm{NB}$ in vitro models. ${ }^{42}$ By contrast, we found three genes from the mTOR pathway to be upregulated by MYCN, which strongly supports the notion that the mTOR/PI3K/ Akt axis is an important feature of MYCN-driven survival capabilities of tumor cells. It could also been shown that interference with this pathway in turn leads to the degradation of MYCN protein in neuroblastoma using small molecule inhibitors of mTOR such as rapamycin or novel drugs such as BEZ-235 currently in clinical development. ${ }^{43,44} \mathrm{We}$ here demonstrated tumor-specific activation of the mTOR pathway in a MYCN-driven neuroblastoma mouse model. Furthermore, we confirmed that neuroblastoma cells are sensitive toward mTOR-directed treatment, in line with a previous report demonstrating MYCN downregulation in neuroblastoma cells upon mTOR inhibition. ${ }^{43}$ Here, we show that especially in cells with overexpression or activation of MYCN mTOR inhibition might be appropriate to effectively block proliferation.

Taken together, we provide first insights into the transcriptomal landscape of $M Y C N$-amplified neuroblastoma using next-generation RNA sequencing. We have identified genes and pathways including coding mRNAs as well as noncoding RNAs associated with MYCN in vivo and in vitro. Our results strongly argue in favor of further evaluation of mTOR inhibitors for the treatment of MYCN-amplified NB.

\section{Acknowledgements}

Part of this work was supported by Deutsche Forschungsgemeinschaft (DFG) within the Collaborative Research Center SFB 876 "Providing Information by Resource-Constrained Analysis," subprojects B1 "Spectra" to S.R. and C1 "DimRed" to A.S. The authors thank Melanie Baumann and Sabine Dreesmann for excellent technical assistance and Harald Stephan for preparing the figures. The authors declare that no conflict of interest has to be reported associated with this manuscript.

\section{References}

1. London WB, Castel V, Monclair T, et al. Clinical and biologic features predictive of survival after relapse of neuroblastoma: a report from the International Neuroblastoma Risk Group project. J Clin Oncol 2011;29:3286-92.

2. Baker DL, Schmidt ML, Cohn SL, et al. Outcome after reduced chemotherapy for intermediate-risk neuroblastoma. N Engl J Med 2010;363:1313-23.

3. Hero B, Simon T, Spitz R, et al. Localized infant neuroblastomas often show spontaneous regression: results of the prospective trials NB95-S and NB97. J Clin Oncol 2008;26: 1504-10.

4. Goto S, Umehara S, Gerbing RB, et al. Histopathology (international neuroblastoma pathology classification) and MYCN status in patients with peripheral neuroblastic tumors: a report from the Children's Cancer Group. Cancer 2001;92:2699-708.

5. Seeger RC, Brodeur GM, Sather H, et al. Association of multiple copies of the N-myc oncogene with rapid progression of neuroblastomas. N Engl J Med 1985;313:1111-6.

6. Molenaar JJ, Koster J, Zwijnenburg DA, et al. Sequencing of neuroblastoma identifies chromothripsis and defects in neuritogenesis genes. Nature 2012;483:589-93.

7. Chen Y, Takita J, Choi YL, et al. Oncogenic mutations of ALK kinase in neuroblastoma. Nature 2008;455:971-4.

8. George RE, Sanda T, Hanna M, et al. Activating mutations in ALK provide a therapeutic target in neuroblastoma. Nature 2008;455:975-8.

9. Janoueix-Lerosey I, Lequin D, Brugieres L, et al. Somatic and germline activating mutations of the ALK kinase receptor in neuroblastoma. Nature 2008;455:967-70.

10. Mosse YP, Laudenslager M, Longo L, et al Identification of ALK as a major familial neuroblastoma predisposition gene. Nature 2008; 455:930-5.

11. Caren H, Abel F, Kogner P, et al. High incidence of DNA mutations and gene amplifications of the ALK gene in advanced sporadic neuroblastoma tumors. Biochem J 2008;416:153-9.
12. Mosse YP, Laudenslager M, Khazi D, et al. Germline PHOX2B mutation in hereditary neuroblastoma. Am J Hum Genet 2004;75:727-30.

13. van Limpt V, Schramm A, van Lakeman A, et al. The Phox $2 \mathrm{~B}$ homeobox gene is mutated in sporadic neuroblastomas. Oncogene 2004;23: 9280-8.

14. Buckley PG, Alcock L, Bryan K, et al. Chromosomal and microRNA expression patterns reveal biologically distinct subgroups of $11 \mathrm{q}-$ neuroblastoma. Clin Cancer Res 2010;16:2971-8.

15. Caren H, Kryh H, Nethander M, et al. High-risk neuroblastoma tumors with $11 \mathrm{q}$-deletion display a poor prognostic, chromosome instability phenotype with later onset. Proc Natl Acad Sci USA 2010;107:4323-8.

16. Schulte JH, Marschall T, Martin M, et al. Deep sequencing reveals differential expression of microRNAs in favorable versus unfavorable neuroblastoma. Nucleic Acids Res 2010;38: 5919-28.

17. Schulte JH, Schowe B, Mestdagh P, et al. Accurate prediction of neuroblastoma outcome based on miRNA expression profiles. Int J Cancer 2010;127:2374-85.

18. Mestdagh P, Bostrom AK, Impens F, et al. The miR-17-92 microRNA cluster regulates multiple components of the TGF-beta pathway in neuroblastoma. Mol Cell 2010;40:762-73.

19. Schulte JH, Horn S, Otto T, et al. MYCN regulates oncogenic MicroRNAs in neuroblastoma. Int J Cancer 2008;122:699-704

20. Ross RA, Spengler BA, Biedler JL. Coordinate morphological and biochemical interconversion of human neuroblastoma cells. J Natl Cancer Inst 1983;71:741-7.

21. Schweigerer L, Breit S, Wenzel A, et al. Augmented MYCN expression advances the malignant phenotype of human neuroblastoma cells. Evidence for induction of autocrine growth activity. Cancer Res 1990;50:4411-6.

22. Schulte JH, Schramm A, Klein-Hitpass L, et al. Microarray analysis reveals differential gene expression patterns and regulation of single target genes contributing to the opposing phenotype of
TrkA- and TrkB-expressing neuroblastomas. Oncogene 2005;24:165-77.

23. Klostermeier UC, Barann M, Wittig M, et al. A tissue-specific landscape of sense/antisense transcription in the mouse intestine. BMC Genom 2011;12:305.

24. Benjamini Y, Hochberg Y. Controlling the false discovery rate-a practical and powerful approach to multiple testing. J R Stat Soc B Met 1995;57:289-300.

25. Storey JD. A direct approach to false discovery rates. J R Stat Soc B 2002;64:479-98.

26. Zeller KI, Jegga AG, Aronow BJ, et al. An integrated database of genes responsive to the Myc oncogenic transcription factor: identification of direct genomic targets. Genome Biol 2003;4:R69.

27. Murphy DM, Buckley PG, Bryan K, et al. Global MYCN transcription factor binding analysis in neuroblastoma reveals association with distinct E-box motifs and regions of DNA hypermethylation. PLoS ONE 2009;4:e8154

28. Marschall T, Rahmann S. MoSDi: motif statistics and discovery. 2009. Available at http:// code.google.com/p/mosdi/.

29. Weiss WA, Aldape K, Mohapatra G, et al. Targeted expression of MYCN causes neuroblastoma in transgenic mice. EMBO J 1997;16:2985-95.

30. Gerlinger M, Rowan AJ, Horswell S, et al. Intratumor heterogeneity and branched evolution revealed by multiregion sequencing. $N$ Engl $J$ Med 2012;366:883-92.

31. Parsons DW, Li M, Zhang X, et al. The genetic landscape of the childhood cancer medulloblastoma. Science 2011;331:435-9.

32. Zhang J, Benavente CA, McEvoy J, et al. A novel retinoblastoma therapy from genomic and epigenetic analyses. Nature 2012;481:329-34.

33. Mestdagh P, Fredlund E, Pattyn F, et al. MYCN/ c-MYC-induced microRNAs repress coding gene networks associated with poor outcome in MYCN/c-MYC-activated tumors. Oncogene 2010; 29:1394-404.

34. Wei JS, Song YK, Durinck S, et al. The MYCN oncogene is a direct target of miR-34a. Oncogene 2008;27:5204-13. 
35. Bernard D, Prasanth KV, Tripathi V, et al. A long nuclear-retained non-coding RNA regulates synaptogenesis by modulating gene expression. EMBO J 2010;29:3082-93.

36. Kogo R, Shimamura T, Mimori K, et al. Long noncoding RNA HOTAIR regulates polycombdependent chromatin modification and is associated with poor prognosis in colorectal cancers. Cancer Res 2011;71:6320-6.

37. Niinuma T, Suzuki H, Nojima M, et al. Upregulation of miR-196a and HOTAIR drive malignant character in gastrointestinal stromal tumors. Cancer Res 2012;72:1126-36.

38. Schramm A, Schulte JH, Klein-Hitpass L, et al. Prediction of clinical outcome and biological characterization of neuroblastoma by expression profiling. Oncogene 2005;24:7902-12.

39. Guo X, Chen QR, Song YK, et al. Exon array analysis reveals neuroblastoma tumors have distinct alternative splicing patterns according to stage and MYCN amplification status. BMC Med Genom 2011;4:35.

40. Qing G, Skuli N, Mayes PA, et al. Combinatorial regulation of neuroblastoma tumor progression by N-Myc and hypoxia inducible factor HIF1alpha. Cancer Res 2010;70:10351-61.

41. Boon K, Caron HN, van Asperen R, et al. N-myc enhances the expression of a large set of genes functioning in ribosome biogenesis and protein synthesis. EMBO J 2001;20:1383-93.
42. Krasnoselsky AL, Whiteford CC, Wei JS, et al. Altered expression of cell cycle genes distinguishes aggressive neuroblastoma. Oncogene 2005;24:1533-41.

43. Johnsen JI, Segerstrom L, Orrego A, et al. Inhibitors of mammalian target of rapamycin downregulate MYCN protein expression and inhibit neuroblastoma growth in vitro and in vivo. Oncogene 2008;27:2910-22.

44. Segerstrom L, Baryawno N, Sveinbjornsson B, et al. Effects of small molecule inhibitors of PI3K/Akt/mTOR signaling on neuroblastoma growth in vitro and in vivo. Int J Cancer 2011; 129:2958-65. 